\title{
TRES TORRES, TRES ARQUETIPOS \& TRES CUERPOS PRISMÁTICOS
}

\section{THREE TOWERS, THREE ARCHETYPES AND THREE PRISMATIC BODIES}

\author{
Julio Alfredo Delgado Rojas ${ }^{1}$ \\ Yannette Díaz Umaña² \\ Mawency Vergel Ortega ${ }^{3}$ \\ Universidad Francisco de Paula Santander.
}

\section{$\begin{array}{llll}4 & 0 & 5 & \text { RESUMEN }\end{array}$}

En la investigación de tipo descriptivo se indaga sobre tres torres arquetípicas de la ciudad de Cúcuta, que se circunscriben en el siglo XX como fecha de construcción. Se consigue identificar una estética particular, que le concede a las tres torres un poder territorial, comunicativo $y$ temporal. Así se estima la torre uno a los pies del templo, como instrumento sonoro; la torre dos sobre el presbítero con tipología moderna 1 Magister en Educación Matemática, Arquitecto, Docente. Filiación: Universidad Francisco de Paula Santander. Correo electrónico: julioalfredo@ufps.edu.co Orcid: https://orcid.org/0000-0001-6944-832X Grupo de investigación Euler- Quetelet

$2 \quad$ Magister en Gestión Urbana, Arquitecta, docente y directora del Departamento de Arquitectura, diseño y Urbanismo. Filiación: Universidad Francisco de Paula Santander. Correo electrónico: yannettedu@ufps.edu.co Orcid: https://orcid.org/0000-0003-4582-1593 Grupo de investigación Grupo de investigación Quetelet

3 Doctora en Educación. Postdoctora en Imaginarios y representaciones sociales. Docente y Directora del Departamento de Matemáticas y Estadística. Filiación: Universidad Francisco de Paula Santander. Correo electrónico: mawency@ufps.edu.co. Orcid: https://orcid.org/0000-00018285-2968 Grupo de investigación Quetelet- grupo de investigación Graunt y el reemplazo de las campanas por un sistema mecanizado de sonido; y la torre tres alejada del cuerpo de la iglesia, sobreponiendo su capacidad de apropiación geofísica y comunicativa. De este modo las 3 torres salvaguardan los conceptos de las estructuras medievales sagradas, haciendo uso de un lenguaje arquitectónico propio a su fecha de construcción. El marcaje, apropiación del territorio, el control del tiempo y la comunicación son facultades que consiguen estos cuerpos primaticos dos cuadrangulares y uno trapezoidal, por lo que se puede decir que son poderosos hitos referenciales de la fe católica en el paisaje urbano de san José de Cúcuta.

\section{PALABRAS CLAVE}

arquitectura sagrada, torres, castrense, geometría. 


\section{ABSTRACT}

Descriptive research investigates three archetypal towers of the city of Cúcuta, which are circumscribed in the 20th century as the date of construction. It is possible to identify a particular aesthetic, which gives the three towers a territorial, communicative and temporal power. Thus the tower one at the foot of the temple is considered as a sound instrument; tower two on the presbytery with modern typology and the replacement of the bells by a mechanized sound system; and tower three away from the body of the church, superimposing its geophysical and communicative appropriation capacity. In this way, the 3 towers safeguard the concepts of medieval sacred structures, making use of an architectural language typical of its construction date. Marking, appropriation of the territory, control of time and communication are faculties that these primatic bodies achieve, two quadrangular and one trapezoidal, so it can be said that they are powerful benchmarks of the Catholic faith in the urban landscape of San José de Cucuta.

\section{KEYWORDS}

sacred architecture, towers, military, geometry.

\section{INTRODUCCION}

Las torres son conocidas como edificios constructivos esbeltos, cuyas dimensiones en planta son mucho menor en comparación a la altura, se puede decir que desde un principio se ha reconocido como la huella en el tiempo en la vida de los pueblos y ciudades hasta el siglo XX (Sanchez y Fernández 2015). El significado espiritual de estas estructuras viene directamente de la Biblia, en donde se presenta varias consideraciones, por ejemplo, relacionarla con la atribución a Dios del resguardo, la confianza y el poder. En el Salmos 61,2-3 habla sobre Dios como una torre cuando dice: "Llévame a la roca que es más alta que yo, porque tú has sido mi refugio y torre fuerte delante del enemigo". De nuevo en Proverbios 18,10 se expresa: "El nombre del Señor es una torre fortificada: el justo corre hacia ella y se pone a salvo".

Las connotaciones bíblicas permiten definir el propósito constructivo de la torre, en el papel territorial y defensivo, así se cita en 2 Crónicas 14:7 "Dijo, pues, a Judá: Edifiquemos estas ciudades y cerquémoslas de murallas con torres, puertas y barras. La tierra es aún nuestra, porque hemos buscado al señor nuestro Dios; le hemos buscado, y él nos ha dado tranquilidad por todas partes. Edificaron, pues, y prosperaron. De la misma manera en 1 Crónicas 27:25 "A cargo de los almacenes del rey estaba Azmavet, hijo de Adiel. Y a cargo de los almacenes en el campo, en las ciudades, en las aldeas y en las torres estaba Jonatán, hijo de Uzías." De cierta manera se equipará el concepto de torre con mojón, la señal clavada en el suelo que sirve para definir el límite de un territorio o de una propiedad. Cuanto mayor sea la altura, mayor marcaje en el territorio, arraigando la apropiación y su proyección en el territorio.

Por otra parte, en las escrituras también se relacionada la solidez de la torre, con las capacidades defensivas en 2 Crónicas 26:9-10 "Uzías edificó además torres en Jerusalén en la puerta del Angulo, en la puerta del Valle y en la esquina de la muralla, y las fortificó. Edificó también torres en el desierto y excavó muchas cisternas, porque tenía mucho ganado, tanto en las tierras bajas como en la llanura." Con este propósito adquiere ciertas tipologías que facilitan el trabajo defensivo y de ataque. La torre se proyecta tanto en los asentamientos urbanos como rurales, las proporciones de la altura le conferían a la torre un lugar privilegiado para mirar, vigilar y comunicarse, así como lo narra Vergel, Delgado \& Díaz (2018).

También se conoce las definiciones dadas en Delgado, Díaz \& Vergel (2019). cuando citan a: 
"[...] Lorenzo (2007) dice que históricamente el uso de la campana para fines religiosos se remonta al siglo VIII, cuando también se relaciona con la construcción de las torres de las iglesias. Igualmente testifica que no hay iglesia sin campanas, por lo que la torre o la espadaña surge con su único objetivo de sostenerlas, generando un hito jerárquico y llamativo en los parajes urbanos y rurales, tal como fue afirmado por Thurston (1907). A la par, este autor señala que a medida que se hizo necesario convocar a los habitantes de la ciudad o aldea a las celebraciones, se construyeron torres y campanas, que fueron aumentando de tamaño, y número para reforzar el sonido." ( $p$ 53)

Por consiguiente, las torres también se vinculan directamente a oficios litúrgicos, tal como sucede en el imaginario urbano de la ciudad de San José de Cúcuta, cuya manifestación física constructiva de la torre sur en su momento participo de la guerra de los mil días, proveyendo un lugar estratégico de defensa y ataque; y como campanario (Díaz, Delgado \& Vergel 2021), con sus cuatro campanas que han presidido las celebraciones litúrgicas y el paso de las horas diarias.

En el proceso constructivo de las ciudades igualmente se identifica el imaginario de la torre como elemento arquitectónico atractivo por cuanto los metros alcanzados despuntando el perfil de las ciudades y estableciendo jerarquías cada vez más competitivas en este sentido (Díaz, Díaz \& Galvis 2021). De este modo "Las torres campanario constituyen uno de los hitos referenciales más poderosos en la memoria visual de una cultura como la nuestra, transida por los signos del cristianismo" (Gómez, 2009, p181)

\section{ANÁLISIS Y DISCUSIÓN}

Observando la arquitectura eclesiástica, se identifica la torre, como la estructura vertical construida en el lateral, frente, atrás o sobre la iglesia, que sobresale de su entorno construido. Bajo esta definición, se seleccionan tres torres construidas en el siglo XX en la ciudad de Cúcuta, por cuanto su disposición respecto al templo cuya estructura representativa se advierten en las variantes propuestas por Sánchez (2009) donde se identifica entre ellas, torre adosada a la iglesia a los pies de la nave central y laterales, la torre situada directamente sobre el ábside o cabecera de la iglesia y la torre desplazada e independiente de la arquitectura del templo. Se asemejan del mismo modo en esta muestra aquellas torres que por su cuerpo geométrico y altura cobran un interés particular en el paisaje urbano.

La primera torre estudiada, corresponde a la torre adosada "a los pies del templo" (Sánchez, 2009, p124), es el caso de las torres de la catedral de San Jose de Cúcuta, ubicada junto al parque principal de la ciudad, cuya estructura independiente de la nave central y laterales conserva la proximidad y estética necesaria para percibirlas como un mismo cuerpo. El origen de esta ubicación podría considerarse dentro de la arquitectura cristiana occidental, en las iglesias otonianas del Siglo VIII (Sanchez, San Jose, y Fernández 2015). Así, el arquetipo de este estilo, presenta en todos los casos reseñados las torres junto a la portada principal del edificio, haciendo alusión a su función protectora. Sanchez (2009) confirma que "En tierras burgalesas la presencia de la torre a los pies debió ser timbre de gloria de los edificios religiosos pues aparecen con gran potencia en los últimos años del gótico" (p.126) 
Estas torres de la catedral en San José de Cúcuta, se encuentran protegiendo y controlando el acceso por sus 3 entradas al costado norte y sur, justo a los pies de la nave central y laterales actuado perceptivamente en la fachada como una muralla. Esta ubicación sumada a la "estética castrense" como lo denomina Vergel, Delgado \& Díaz (2020), explica la intención proyectista al querer proteger el templo en momentos de poca estabilidad económica, política y social en Colombia con la guerra de los mil días hacia 1900, descrita por Díaz, Delgado \& Vergel (2021). De esta forma Dimanuel (2006) refiere que con frecuencia las aberturas se daban hacia la parte superior, los pisos bajos el refugio y los últimos pisos como puesto de guardia.

Esto explica que cada una de las torres están proyectadas sobre una planta casi cuadrada semioctogonal con acabado en ladrillo al interior, en un área aproximada en base de cada una de $49 \mathrm{~m} 2$ y una altura de $35 \mathrm{~m}$ de altura. Sus tramos son diferentes alturas configurando una torre con niveles internos culminando la composición arquitectónica con cúpula, linterna, copulín y cruz. Para Delgado, Díaz \& Vergel (2019):

"Su organización interna, aun cuando en el interior parezca una caja vacía, proyecta soluciones estructurales y funcionales adecuadas a su fin de ser audible y visible en el territorio, como ocurre en algunos campanarios de estilo románico" (p.57)

En este sentido Vergel, Delgado \& Díaz (2018) afirman que se estableció como la construcción más alta de la ciudad a principios del siglo $X X$, además de ser el mayor instrumento sonoro de la ciudad, permitiendo como una caja de resonancia, lograda con su forma prismática cuadrangular, donde la salida del sonido se hace a través de sus vanos románicos a cada lado. Al mismo tiempo la escalera en túnel insertada al interior de la base de la torre permite el ascenso del campanero y manipulación de las campanas por medio de sogas atadas al badajo, lo que hace de su arquitectura extrañamente oculta y laberíntica una torre ${ }^{4}$ con influencia medieval, tal como lo relaciona Vergel, Díaz \& Delgado (2021), con las inspiraciones estilísticas de la catedral.

En cuanto al papel comunicativo de la torre, es posible solo desde la torre norte dotada de campanas, con:

"[...] la Campana Santa Madre
de Dios, Campana Mayor
Sagrado Corazón de Jesús,
Campana Menor San Luis, y
la Campana Media, llamada
para efectos de este estudio
San José, que se escuchan
con claridad en una extensión
considerable de la ciudad."
(Delgado, Díaz \& Vergel 2019,
p.54)

En efecto los toques se configuran como marcas sonoras que dan identidad al paisaje arquitectónico como se expresa en Díaz, Delgado \& Vergel (2021). Al respecto De Bustos (2021) dice que "los estatutos capitulares en los que se explicaba cuándo tocar y cómo, constituyendo así completos sistemas de comunicación" pues los toques son un arte distintivo con lenguaje propio, tradición, que, a los ojos de esta autora, significan al mismo tiempo un control del tiempo, al denotar cada toque como una señal de llamado a las actividades litúrgicas, sociales, culturales 0 simplemente las horas del día. (imagen 1)

\footnotetext{
$4 \quad$ En este caso solo la torre norte es quien contiene las campanas y el pasaje de asenso embebido en su muro. "Se da en muros excepcionalmente anchos, en los cuales se insertan las escaleras a modo de corredor comunicando los diferentes pisos de la torre. En la provincia de Burgos se detecta por primera vez en la torre de Doña Urraca de Covarrubias, construcción probable del siglo X" (Sánchez, 2009, p.129)
} 
Imagen 1. Fotografía, corte y forma geométrica de las torres de la catedral de San José de Cúcuta.

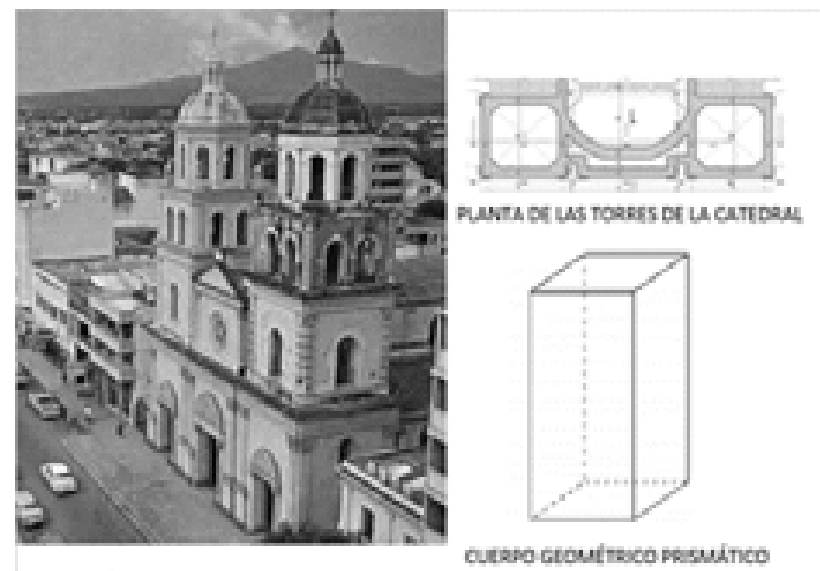

Fuente: elaboración propia.

La segunda torre propuesta para este reconocimiento, se da sobre la conjunción de iglesia y torre en una misma estructura construida, la torre situada directamente sobre el ábside o cabecera de la iglesia. Sánchez (2009) sostiene que "Nada hay en la esencia de la doctrina cristiana que obligue a la ubicación de la torre en un lugar determinado, como tampoco se prescribe la forma que ha de tener la iglesia." (p.124). Sin embargo, se observa a lo largo de la tradición constructiva de los templos católicos, que uno de los lugares predilectos será el presbiterio o cabecera del templo, valiéndose de la disposición del ábside (Sánchez 2009). En este orden de ideas, la iglesia de nuestra señora del Carmelitas, se identifica por tener su torre directamente sobre el área donde comúnmente estaría el ábside, el presbítero e inclusive la cúpula de modo que se unen los 3 elementos

simbólicos (Díaz, Delgado \& Vergel 2020) muy reconocidos en un mismo cuerpo geométrico de la torre.

Para Díaz, Delgado \& Vergel (2021) en la composición general de esta iglesia, se establece un orden semiradial de la cubierta la cual se origina encima del presbítero, aprovechando el contrafuerte donde se dispone un arco de descarga, que permite que todo el peso de la torre y de la cubierta se traslade a ambos lados de las paredes de la torre generando un vano. Esto se comprende al examinar la base de la torre abierta en uno de los lados, dando lugar a las jambas (muros laterales de soporte) entre los cuales se ubica el altar. De esta manera la base de la torre es trapezoidal cuya área es $43 \mathrm{~m} 2$ ordenando un prisma de base de trapecio, que a medida que se levanta sobre el suelo a 21.5 metros de altura disminuye su sección en la cúspide.

Este cuerpo geométrico exteriormente liso, hermético, y vacío al interior, en su trasfondo continua el modelo románico dada la ubicación sobre el presbítero, para introducirse en la conceptualización moderna, de la simplificación de las formas y el rechazo al ornamento típico de la modernidad (Díaz, Delgado \& Vergel 2021) en coherencia con la fecha de construcción en 1969, que corresponde con este momento estilístico (Vergel, Delgado \& Díaz 2020). Así la estructura de la torre es una caja vacía, no posee campanario, acogiéndose al Concilio Vaticano II, que influyo en la desaparición de los campaneros hacia la década de los sesenta, atendiendo los sistemas mecanizados de sonidos (Delgado, Díaz, y Vergel 2019), a cambio un parlante eléctrico en su ápice, para suplir la necesidad comunicativa de la Iglesia.

Otra propiedad adquirida por la torre gracias a su geometría y pequeños vanos que abren los muros lisos del prisma con la cruz, vista desde su fachada principal, garantiza la entrada de haces lumínicos que alcanzan a penetrar la torre 
generando un efecto fenomenológico sobre el altar, como tradicionalmente de forma análoga lo haría los vitrales del tambor al presbítero (Díaz, Vergel \& Delgado 2020). También se halla con este detalle utilidad como respiradero, de allí la referencia histórica, es el uso de las saeteras, pequeñas y estrechas aberturas en el grosor de los muros, habitualmente vistas en las superficies altas de las torres. (imagen 2)

Imagen 1. Fotografía, corte y forma geométrica de la torre de la iglesia de nuestra Señora del Carmelitas.
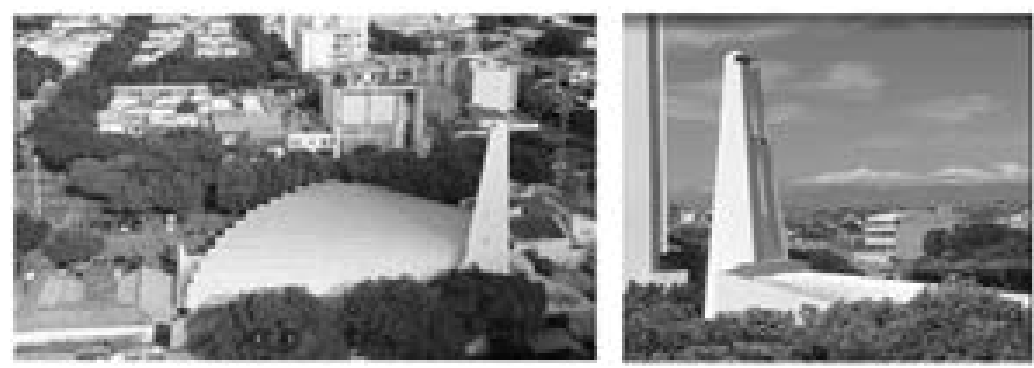

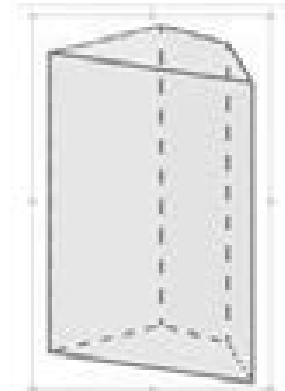

cutrosecout reco rawnince

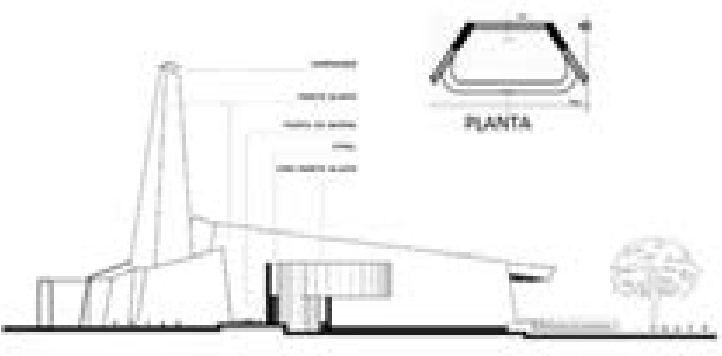

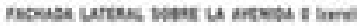

Fuente: elaboración propia con corte de los estudiantes Angie Carrillo, Keyla Vega, Carlos Andrés Conde.

Con estos detalles se podría afirmar que la torre con su estética particular de prisma trapezoidal abandona su función primaria de campanario, sin hacerlo en lo comunicativo, adquiriendo un rol constructivo, estructural, morfológico y simbólico en la iglesia de nuestra señora del Carmelitas. Es de resaltar en este tipo de soluciones, la habilidad de comprensión y salvaguardia de conceptos de las estructuras medievales sagradas, en la proyección de esta torre que ostenta la novedosa concepción moderna apropiándose del perfil de la ciudad, como una marca territorial de la consagración del espacio urbano.

La tercera torre como prototipo analizado es la de la iglesia de Maria Auxiliadora cuya construcción inicio en 1951 en el barrio popular (La Opinión 2018). Se distingue esta torre, al igual que la del Carmelitas por una sintonía compositiva con la modernidad. La variante que la hace diferente de las torres de la catedral y la iglesia de nuestra Señora del Carmelitas, es su separación con el cuerpo del templo. En este arquetipo Sánchez (2009) confirma que "No es preceptivo que la 
torre esté adosada físicamente a la fábrica de la iglesia. Se dan casos en los que la torre dista unos metros del edificio de culto." (p. 123) es decir aquí el templo se ha construido apartado de la torre, lo que resulta poco habitual.

De forma que esta torre de la iglesia de Maria Auxiliadora de 30 metros de altura, se configura en una base cuadrada de $25 \mathrm{~m} 2$ que al elevarse en altura forma el prisma cuadrangular. Se conoce por La opinión (2021) que "[...] su emblemática torre del reloj, que hasta los años setenta fue considerada la más alta de Cúcuta. $Y$ si bien ahora no conserva el título, aún es visible desde otros barrios cercanos, e innegable es girarse en su dirección cada vez que el repicar de las campanas anuncia la eucaristía." por lo que se puede dar constancia de su función comunicativa y territorial.
Otra característica de esta esbelta estructura de hormigón abierta, es que la escalera que recorre su interior queda completamente al descubierto, por lo que se puede apreciar sus más de cuatro niveles internos. Esta solución en la envoltura parece controvertir el lenguaje discreto y hermético de las saeteras, logrado con el uso de delgados listones que conforman dilataciones verticales en todo el cuerpo de la torre, lo que descubre completamente su interior. Esta tipología cambia el paradigma de la torre en su función originaria del propósito militar expuesto por (representaciones), revelando que el contexto temporal de su construcción de la torre en Maria Auxiliadora no hacia vital el papel defensivo, sino geofísico y comunicativo. (imagen 3)

Imagen 1. Fotografía, cortes y forma geométrica de la torre de la iglesia de nuestra Señora del Carmelitas.
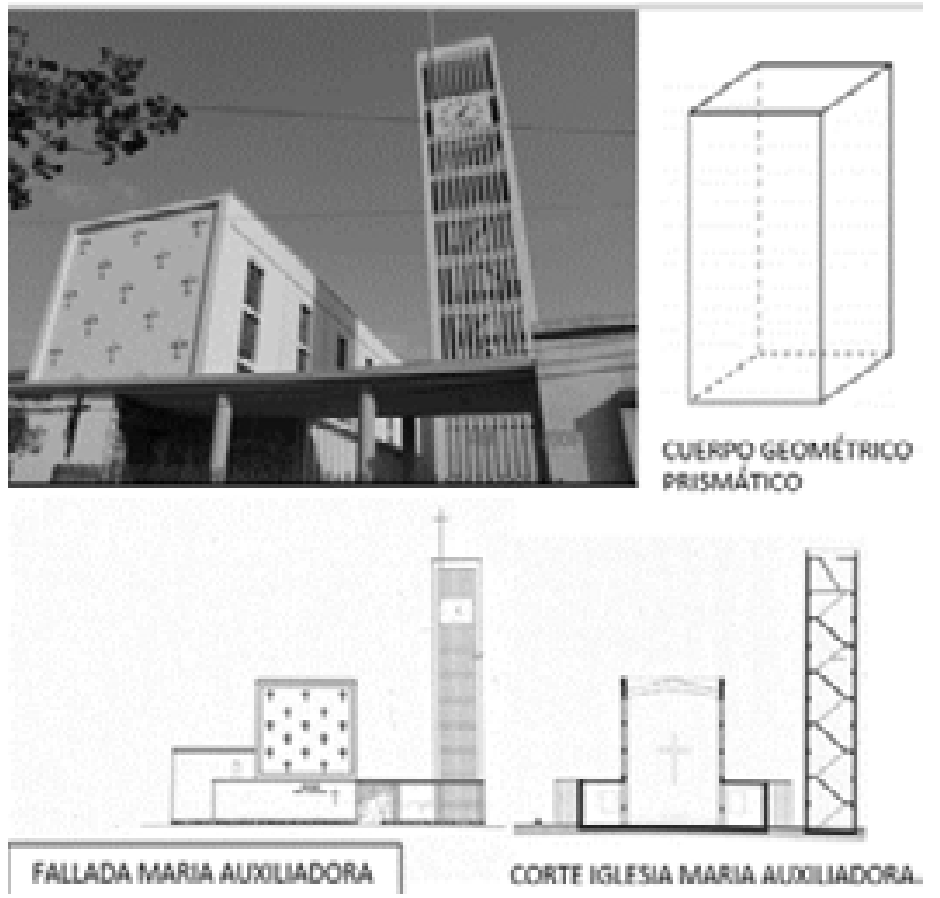

Fuente: elaboración propia con cortes de los estudiantes Manuel Guardiola y Jose Quinceno. 
Esto último se manifiesta en la medida que se dispone de forma muy visible en el skyline del perfil urbano, como mojón del espacio y tiempo en su cercanía con el acceso de todo el complejo. En esta nueva versión que de cierto modo se conserva el simbolismo territorial y se desvincula de la naturaleza defensiva de la torre, otros elementos como el campanario refuerzan el papel comunicativo propio de esta 3 torre. También la aparición del reloj cuadrado se distingue, como un detalle estilístico con remembranza al rosetón del gótico. Otro aspecto significativo es el comentado anteriormente por De Bustos (2021) al afirmar que desde las torres se controlaba el tiempo "Con la llegada de los relojes mecánicos en el siglo XIV, las catedrales no quisieron renunciar al control sobre el tiempo" (p.1).

Este detalle vale la pena examinarlo y contextualizarlo en Colombia, con la llegada de los primeros relojes públicos que aparecieron y se instalaron en las torres de las iglesias, probablemente a comienzos del siglo XIX (EI tiempo 2014). Este medio no confirma con exactitud cuál fue el primero, pero asocia estos mecanismos requeridos por las autoridades civiles y pobladores como emblemáticas maquinarias importadas de Estados Unidos, Europa y hacia la década de los 50 se instalaron los de fabricación nacional, producidos por la firma Talleres Robledo de Medellín (El tiempo 2009), lo que convierte a estos objetos en elementos de valor patrimonial por cuanto su valor artístico y los esfuerzos en las gestiones para adquirir, desplazar e instalar estas maquinarias de uso público.

En concreto se tiene que la torre de la parroquia Maria Auxiliadora consolida el arquetipo moderno, llevando consigo elementos básicos como la geometría prismática cuadrangular de forma esbelta, sencilla, desnuda y sonora. La incorporación del reloj, como un mecanismo apreciable que sirve para medir el tiempo tal como Bustos (2021) refiere el uso de las torres con fines eclesiásticos, la hacen especialmente reconocible por los habitantes de la ciudad, sobreponiendo su capacidad de apropiación geofísica y comunicativa.

\section{CONCLUSIÓN}

Las torres se vinculan históricamente a su función defensiva, comunicativa y litúrgica, tal como sucede en el imaginario urbano de la ciudad de San José de Cúcuta. Al analizar las tres torres seleccionadas por cuanto su ubicación, cuerpo geométrico y fecha de construcción determinada en el siglo XX. Se descubre además la necesidad en el control sobre el tiempo, la adaptación estilística con las que fueron proyectadas dejando una huella en el tiempo. De esta manera tres arquetipos se distinguen por cuanto su disposición de la torre con respecto al templo: la torre adosada a los pies de la iglesia, la torre situada directamente sobre el ábside o cabecera de la iglesia y la torre desplazada e independiente de la arquitectura del templo.

De ahí fueron seleccionadas la catedral de San José de Cúcuta, la iglesia de nuestra Señora del Carmelitas y la iglesia de Maria Auxiliadora conformando una estética particular, que le concede a la torre un poder territorial, comunicativo y temporal. De esta forma la torre norte de la catedral de San José de Cúcuta es considerada un gran instrumento sonoro con estilo castrense. La torre de la iglesia de nuestra Señora del Carmelitas logra mostrar soluciones modernas con la sustitución de las campanas por un sistema mecanizado de sonido, apropiándose del paisaje moderno sonoro, como una marca territorial y comunicativa de la consagración del espacio urbano.

Por último, la torre de la parroquia Maria Auxiliadora logra arraigar los factores estilísticos modernos sobreponiendo su capacidad de apropiación geofísica y comunicativa, con 
un simple cuerpo prismático cuadrangular como estructura. De este modo las 3 torres salvaguardan los conceptos de las estructuras medievales sagradas, haciendo uso de un lenguaje arquitectónico propio a su fecha de construcción. El marcaje, apropiación del territorio, el control del tiempo y la comunicación son facultades por las cuales se puede decir que son poderosos hitos referenciales de la fe católica en el paisaje urbano de san José de Cúcuta.

\section{REFERENCIA BIBLIOGRÁFICAS.}

De Bustos, Rosa (2021). Cuando las catedrales eran el hombre del tiempo. La vanguardia. https://www. lavanguardia.com/historiayvida/ propuestas/20201028/34079/ catedrales-hombre.html

Delgado Rojas, J. A.., Díaz Umaña, Y.., \& Vergel Ortega, M.. (2019). El paisaje de la Catedral de San José de Cúcuta. Revista Logos, Ciencia \& Tecnología, Vol. 11, No. 1, Enero Marzo. https://scholar.google.com/ citations?view_op=view_citation \&hl=e s\&user=carfqowAAAAJ\&citation_for view=carfqowAAAAJ:d1gkVwhDpl0C

Dimanuel Mercedes. (2006) Estructuras $y$ elementos militares en iglesias fortificadas medievales españolas. Anales de Historia del Arte 2006, 16, 79-102. http://revistas.ucm.es/ index.php/ANHA/article/download/ ANHA0606110079A/31089

Díaz Umaña, Y.., Delgado Rojas, J. A.., \& Vergel Ortega, M. (2021). Representaciones socioespaciales, en la memoria histórica de la catedral y su entorno urbano en San José de Cúcuta. Revista Boletín Redipe, 10(4), 376-387. https://doi. org/10.36260/rbr.v10i4.1277

Díaz, Y., Delgado, J. \& Vergel, M. (2021). Estructura como envolvente en la iglesia nuestra Señora del Carmen. Revista Boletín Redipe vol. 10 no. 8

Díaz-Umaña, Y., Delgado-Rojas, J. A.., \& Vergel-Ortega, M. (2021). La geometría basada en la malla como mecanismo compositivo de Villa Savoye. Eco Matemático, 12(1). https://doi. org/10.22463/17948231.3070

Díaz Umaña, Y.., Delgado Rojas, J. A., \& Vergel Ortega, M.. (2020). La geometría constructiva del cimborrio de la catedral de San José de Cúcuta. Revista Boletín Redipe, 9(12), 294-299. https://doi. org/10.36260/rbr.v9i12.1156

Díaz-Umaña, Y., Díaz- Fuentes, X. \& GalvisCenturión, R. (2021). 35 proyectos de arquitectura contemporánea. Materia forma \& contexto. ECOE ediciones. Bogotá.

Díaz, Yannette, Vergel, Mawency y Delgado Julio (2021). Entre las torres de San José. Aproximaciones al arte, arquitectura y geometría de la catedral. ECOE Editorial, Bogotá.

Díaz, Yannette, Vergel, Mawency y Delgado Julio (2021). El espíritu de la luz. Iconografías y geometrías de la arquitectura en la catedral de san Jose de Cúcuta. ECOE Editorial, Bogotá. 
Díaz-Umaña, Y.., Vergel-Ortega, M. . ., \& Delgado-Rojas, J. A. . (2020). Modelo geométrico y arquitectónico de la cúpula mayor en San José de Cúcuta. Revista Boletín Redipe, 9(3), 160-166. https:// doi.org/10.36260/rbr.v9i3.941

El tiempo. (2014). Los relojes más emblemáticos de Bogotá. 13 de 02014 https://www. eltiempo.com/archivo/documento/CMS13985003

El tiempo (2009). Inventario de los relojes que hay en edificaciones de Bogotá hará Instituto Distrital de Patrimonio. 5 septiembre https://www.eltiempo.com/ archivo/documento/CMS-6029887

Gómez, Javier (2009). Torres campanario. Entre el templo clásico y la iglesia gótica. Apuntes de Historia del Arte.p179 en https://www.docsity.com/es/torrescampanario-entre-el-templo-clasico-yla-iglesia-gotica/4565212/

La opinión. (2021).El Popular: la historia detrás del primer barrio de clase media de Cúcuta.12 de febrero https://www. laopinion.com.co/comunidad/elpopular-la-historia-detras-del-primerbarrio-de-clase-media-de-cucuta

La Opinión (2018). Bendición de la construcción de la iglesia María Auxiliadora. 3 de agosto. https://www.laopinion. com.co/memorias/bendicion-de-laconstruccion-de-la-iglesia-mariaauxiliadora

Sanchez, Jose; San Jose, Jesús y Fernández, Martin. (2015). Ocho torres: análisis sobre la evolución de campanarios del siglo $\mathrm{XVI}$ en la provincia de burgos. Editorial: Universidad de Valladolid, España,
Sánchez, José (2009). Torres defensivas y campanarios de iglesia: villaescusa de roa en la ribera del Duero. Universidad de Valladolid https://dialnet.unirioja.es/ descarga/articulo/3296441.pdf

Vergel-Ortega, M; Delgado Rojas, J \& Díaz Umaña, Y (2018). Cathedral bell's San José de Cúcuta: Heritage and acoustics. Journal of Physics: Conference Series, Volume 1329, XIV Applied Mathematics Meeting and XI Statistics Meeting 7-8 December 2018, San José de Cucuta, Colombia

https://iopscience.iop.org/ article/10.1088/1742-6596/

\section{9/1/012004/meta}

Vergel Ortega, M.., Delgado Rojas, J. A.., \& Díaz Umaña, Y. (2020). Estudio iconográfico y geométrico del vitral de San José en la catedral de Cúcuta. Revista Boletín Redipe, 9(6), 119-133. https://doi. org/10.36260/rbr.v9i6.1006

Vergel Ortega, M.., Díaz Umaña, Y. \& Delgado Rojas, J. A (2021). Dependencia geométrica en la composición del vitral del Espíritu Santo. un estudio iconográfico y arquitectónico en la catedral de san José de Cúcuta. Revista Boletín Redipe vol. 10 no. 8

Vergel Ortega, M.., Delgado Rojas, J. A.., \& Díaz Umaña, Y. (2020). Dimensión histórica de la red ferroviaria, su impacto económico y urbano en San José de Cúcuta. Revista Boletín Redipe, 9(6), 183-191. https://doi.org/10.36260/rbr. v9i6.1011 
Vergel Ortega, M., Díaz Umaña, Y.., \& Delgado

Rojas, J. A. (2020). Luz, símbolo y revelaciones a través del papiro de Jeremías y la presencia de la virgen María en la catedral de San José de Cúcuta. Revista Boletín Redipe, 9(11), 272-281. https://doi.org/10.36260/rbr. v9i11.1130 